\title{
Understanding the Interdependencies Between Composition, Microstructure, and Continuum Variables and Their Influence on the Fracture Toughness of $\alpha / \beta$-Processed Ti-6Al-4V
}

\begin{abstract}
P.C. COLLINS, S. KODURI, V. DIXIT, and H.L. FRASER
The fracture toughness of a material depends upon the material's composition and microstructure, as well as other material properties operating at the continuum level. The interrelationships between these variables are complex, and thus difficult to interpret, especially in multi-component, multi-phase ductile engineering alloys such as $\alpha / \beta$-processed Ti-6Al-4V (nominal composition, wt pct). Neural networks have been used to elucidate how variables such as composition and microstructure influence the fracture toughness directly (i.e., via a crack initiation or propagation mechanism) - and independent of the influence of the same variables influence on the yield strength and plasticity of the material. The variables included in the models and analysis include (i) alloy composition, specifically, Al, V, O, and Fe; (ii) materials microstructure, including phase fractions and average sizes of key microstructural features; (iii) the yield strength and reduction in area obtained from uniaxial tensile tests; and (iv) an assessment of the degree to which plane strain conditions were satisfied by including a factor related to the plane strain thickness. Once trained, virtual experiments have been conducted which permit the determination of each variable's functional dependency on the resulting fracture toughness. Given that the database includes both $\boldsymbol{K}_{\mathbf{1 C}}$ and $\boldsymbol{K}_{\boldsymbol{Q}}$ values, as well as the in-plane component of the stress state of the crack tip, it is possible to quantitatively assess the effect of sample thickness on $\boldsymbol{K}_{\boldsymbol{Q}}$ and the degree to which the $\boldsymbol{K}_{\boldsymbol{Q}}$ and $\boldsymbol{K}_{\boldsymbol{1}}$ values may vary. These interpretations drawn by comparing multiple neural networks have a significant impact on the general understanding of how the microstructure influences the fracture toughness in ductile materials, as well as an ability to predict the fracture toughness of $\alpha / \beta$-processed Ti-6Al-4V.
\end{abstract}

https://doi.org/10.1007/s11661-017-4443-9

(C) The Minerals, Metals \& Materials Society and ASM International 2018

\section{INTRODUCTION}

THE fracture toughness $(\boldsymbol{K})$ of a material describes how the material will respond when subjected to a load with a critical flaw size. This property, while important for the design engineer, is an often ill-studied problem, costly to measure, and is exceptionally difficult to predict. While progress has been made to predict the yield strength and fatigue properties of engineering

P.C. COLLINS is with the Department of Materials Science and Engineering, Iowa State University, Ames, IA 50011 and also with the Center for Advanced Non-Ferrous Structural Alloys, an NSF I/ UCRC, Ames, IA 50011. Contact e-mail: pcollins@iastate.edu S. KODURI and V. DIXIT are with Intel Corporation, Hillsboro, OR 97124. H.L. FRASER is with the Department of Materials Science and Engineering, The Center for the Accelerated Maturation of Materials, The Ohio State University, Columbus, OH 43210.

Manuscript submitted December 6, 2016.

Article published online January 4, 2018 alloys given a specific composition and microstructure (including titanium based alloys ${ }^{[1-6]}$ ), and while fundamental relationships between composition and slip mechanisms have been determined, ${ }^{\left[{ }^{-11]}\right.}$ the prediction of fracture toughness based upon composition and microstructure is far less mature. In other words, there is a "knowledge gap" or "predictability gap" when it comes to fracture toughness. This is especially true for ductile materials, such as $\alpha / \beta$-processed Ti-6Al-4V.

The origin of this "predictability gap" may be attributed to the complex manner by which fracture occurs (i.e., by which cracks propagate). The area immediately in front of a crack tip is subjected to local stresses which may exceed the tensile strength of the material $\left(\sigma_{\mathrm{ys}}=f\right.$ (composition, microstructure $\left.)\right)$. The specimen geometry and dimensions, initial flaw size, state of stress at the crack tip, and the response of the material to the concentrated stress and crack propagation all contribute to the fracture toughness. The degree to which these relationships are known varies significantly. For example, there is a reasonable level of 
maturity with regard to the understanding of the relationships between applied stress, basic sample geometries, and initial crack shapes/sizes and the resulting fracture toughness. Conversely, there is generally a dearth of legacy knowledge regarding the influence of the individual continuum material parameters (e.g., yield strength and the state of stress at the crack tip, $\sigma_{z}$ ) on the measured toughness. This knowledge gap includes (generally) any understanding of how the microstructure responds to the presence of a crack under load, including crack tip opening and growth and the development of damage (e.g., secondary microcracks) in the immediate vicinity of the primary crack. This knowledge asymmetry is shown schematically in Figure 1 with the well-established connection between geometry and crack size and the material property shown with a solid line. In a similar fashion, the various interconnected material variables are shown with dotted lines to indicate the lack of understanding of their interrelationships and contribution to the material response, $\boldsymbol{K}$.

Beyond the variables included directly in fracture mechanics, one relationship has been fairly well established, namely the inverse relationship between yield strength and fracture toughness (see Figure 2). The data in this schematic diagram have been compiled from a variety of sources and for a range of Ti-based alloys. Similar plots have guided alloy selection and fracture toughness knowledge and are shown throughout the literature [e.g., Reference 12]. However, owing to the difficult nature of the interpretation of the material response, there have been few efforts to move beyond this rudimentary observation and establish a mechanistic understanding of the influence of microstructure on the fracture of ductile multi-phase materials in general and for Ti-based alloys specifically. The notable exceptions for ductile multi-phase materials are the seminal work of Ritchie and Thompson, ${ }^{[13]}$ and the more recent computational work by Osovski et al. ${ }^{[14]}$ and others. ${ }^{[15]}$

An additional barrier exists when attempting to develop a micromechanistic understanding of fracture. This barrier is related to the geometry-dependent variability of the local stress state at the crack tip. While Figure 1 appropriately labels the material response as $\boldsymbol{K}$, the specific $\boldsymbol{K}$ that is determined is a function of the stress state at the crack tip, which in turn is a function of both sample geometry and the local yield strength $\left(\sigma_{\mathrm{ys}}\right)$. It is well known that the stress state at the crack tip, specifically the in-plane stress parallel to the crack front $\left(\sigma_{z}\right)$, can result in different fracture toughness values based on variations in sample thickness. This variability is depicted in Figure 3, where the $\boldsymbol{K}$ increases with decreasing sample thickness, due to the pronounced role of the plane stress condition within the sample. As can be seen, the radius of the plastic zone size $\left(r_{y}\right)$ also plays a role. This size is related directly to the square of the ratio between the fracture toughness and the yield strength of the material in which the plastic zone resides, which can range from several microns to several millimeters, depending upon both $\sigma_{\mathrm{ys}}$ and $\boldsymbol{K}_{\mathbf{1}} \boldsymbol{C}$. Experimentally, it is often challenging to ensure that all tested samples result in a $\boldsymbol{K}_{\mathbf{1} \boldsymbol{C}}$ value, rather than an apparent toughness, or $\boldsymbol{K}_{\boldsymbol{Q}}$ value. This is because the threshold of specimen size between plane strain and plane stress, and therefore between $\boldsymbol{K}_{\boldsymbol{Q}}$ and $\boldsymbol{K}_{\mathbf{1}}$, is largely dependent upon the composition and the microstructure through their influence on not only the plastic zone size $\left(r_{\mathrm{y}}\right)$ but also on the tensile properties and toughness (i.e., $\left.r_{\mathrm{y}} \propto\left(K_{1 C} / \sigma_{\mathrm{ys}}\right)^{2}\right)$. Further complicating the problem for Ti-based alloys is ensuring experimentally that the heat treatment results in an average microstructure that is uniform over a length scale sufficient to measure a valid $\boldsymbol{K}_{\mathbf{1} \boldsymbol{C}}$. For example, in this work, each coupon is thicker than $25 \mathrm{~mm}$, yet not all have resulted in a valid $\boldsymbol{K}_{1 C}$.

These numerous challenges have severely limited the efforts to conduct studies which detail the role of microstructure on toughness in ductile materials. The available data describing the role of microstructural features and composition on toughness in Ti-6Al-4V provide a qualitative description (e.g., $+/-/ 0)$ of variables which influence fracture toughness, but such work does not directly distinguish whether such variables govern toughness by influencing the continuum (e.g., yield strength) or by determining the micromechanisms (e.g., crack path). ${ }^{[16,17]}$ Notably, the seminal work of Ritchie and Thompson has clearly demonstrated not only the importance of considering both macroscopic (continuum) and microscopic contributions to the fracture toughness in some materials (not Ti-based), but also that the two are interrelated and must be considered together when trying to develop an understanding of crack initiation and crack growth in ductile materials. ${ }^{[13]}$ Other researchers have focused on materials where there are clear microstructural features responsible for crack/void nucleation such as hard intermetallics along grain boundaries. While other authors have followed an approach similar to that of Ritchie and Thompson, most have worked to apply continuum theories to microstructural features. ${ }^{[18,19]}$ Such efforts have, by necessity, relied on unverifiable assumptions regarding the properties of individual phases, without considering the complex interrelationship between the phases, or even of variations in interfacial energy or the mechanical constraint provided by distributions of local phases. By adopting an approach that consists of a combination of neural network models and in-depth characterization, it is possible to analyze and interpret both the continuum and micromechanistic contributions simultaneously. For example (and as will be shown here), it is possible to assess whether a given microstructural feature contributes to a continuum variable (i.e., yield strength), a micromechanistic detail (i.e., microcracking or void nucleation), or both.

There have been recent efforts to investigate such interrelationships using neural network approaches. Such a modeling approach has been developed by the authors for the prediction of tensile properties in both $\beta$ and $\alpha / \beta$-processed Ti-6Al-4V. ${ }^{[1-4,20]}$ These research efforts have used models incorporating Bayesian statistics, ${ }^{[21,22]}$ and include metrics for microstructural features which have been quantified using stereological methods, as described elsewhere. ${ }^{[23]}$ Following training 


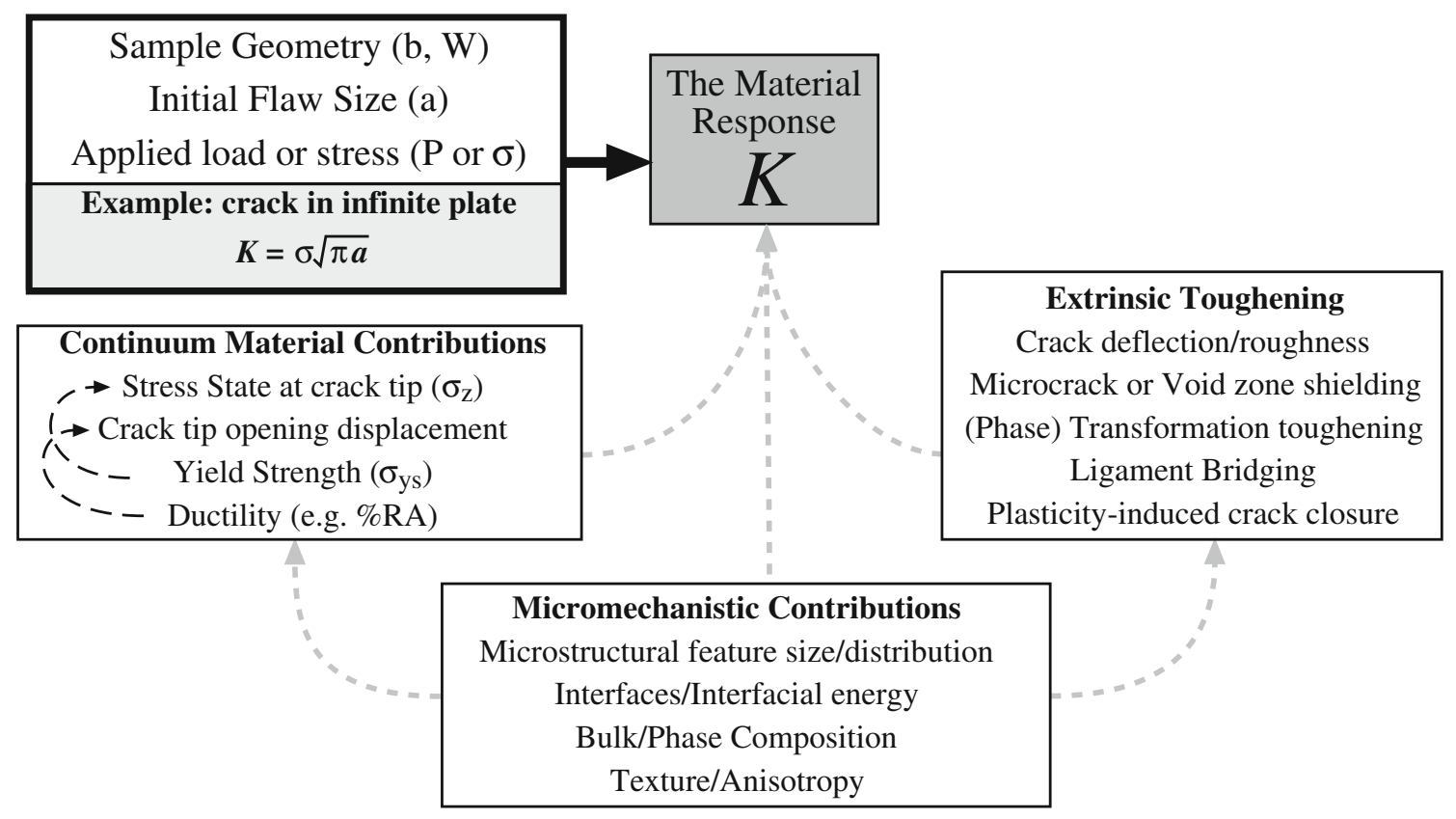

Fig. 1-Schematic of the complexity involved in deriving fundamental details of the fracture toughness problem.

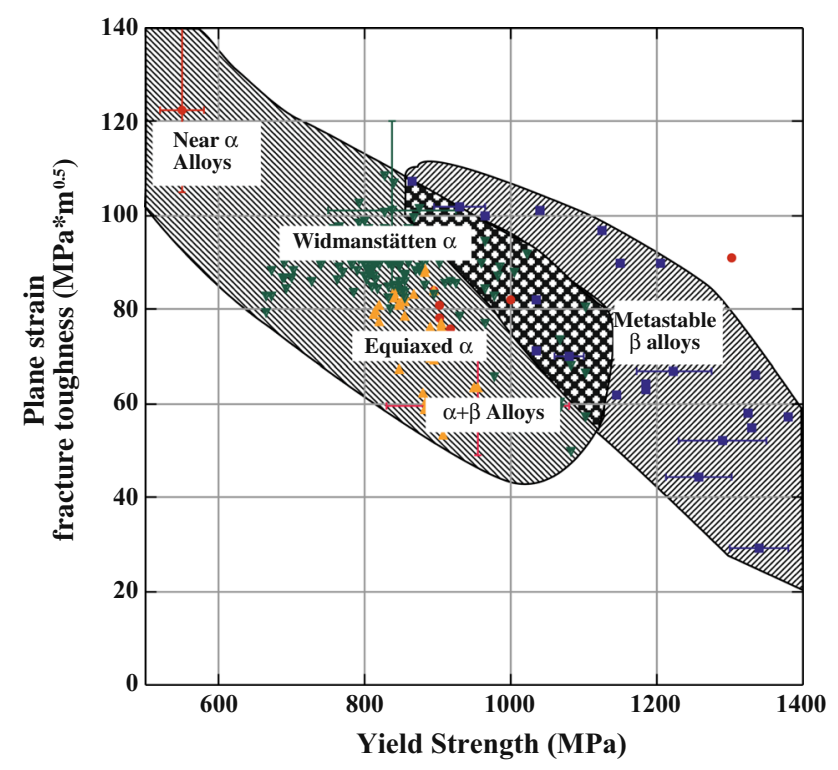

Fig. 2-Inverse relationship between fracture toughness and yield strength for Ti-alloys. The data have been gathered from the research of the authors and that published ${ }^{[25]}$ for the alloys Ti-6-2222S, Ti-6-4, CP Ti, Ti-6242, Ti-5Al-2.5Sn, Ti-8Al-1Mo-1V, beta III, beta C, Ti-10-2-3, Ti-13V-11Cr-3Al, Ti-15V-3Al-3Cr-3Sn, Ti-21S (form similar to that presented in Ref. [12]).

and testing, the models for the interpolative prediction of tensile properties have resulted in differences of less than \pm 2.5 pct from experimentally measured values for both yield strength and ultimate tensile strength. ${ }^{[20]}$ Considering the extended range of tensile properties possible in Ti-6Al-4V (e.g., 725 to $930 \mathrm{MPa}$ for yield strength), a prediction with an associated uncertainty of



Fig. 3-Schematic plot of stress intensity factor $v s$. the ratio of plastic zone size and specimen thickness, where large values of specimen thickness generally correspond to plane strain conditions, whereas, conversely, smaller thicknesses correspond to plane stress.

$\pm 2.5 \mathrm{pct}$ error is quite remarkable. Given the success in applying neural networks for yield strength, the separation of the continuum and micromechanistic contributions of the material to the fracture toughness may be possible using a similar approach.

An additional advantage afforded by neural networks is the potential to conduct virtual experiments. Such experiments, described in detail elsewhere ${ }^{[1-3,20]}$ allow for a single variable (e.g., a microstructural feature, continuum variable, or solute species) to be varied, while the other variables are set to single values. These experiments can be used to visualize and make 
interpretations on a complex, $n$-variable response surface (here, fracture toughness). While such results have been successful in the prediction of tensile properties, the application of neural network models to the prediction of fracture toughness is expected to be somewhat more difficult, and hence, the results potentially less accurate. This is due to the complicated manner in which the material responds to the application of a load in the presence of a flaw. Indeed, the material response and the absence of a sufficient published legacy understanding of the influence of microstructure on fracture might reasonably be expected to present a challenge when verifying the results.

An additional consequence of the models that are developed within this paper is the ability not only to include a factor for the estimation of the thickness of the plane strain region of the specimen, but also to predict a first approximation for the expected plane strain thickness without necessarily knowing the yield strength of the material. The direct inclusion of the plane strain region is important for two reasons. Firstly, its inclusion allows a direct analysis of $\boldsymbol{K}_{\boldsymbol{Q}}$ as a function of thickness, and the comparison of $\boldsymbol{K}_{Q}(\boldsymbol{t})$ with valid $\boldsymbol{K}_{\mathbf{1} \boldsymbol{C}}$ values. This is because the rules-based model not only "scales" the $\boldsymbol{K}_{\boldsymbol{Q}}$ 's to reflect values closer to their $\boldsymbol{K}_{1 C}$ if plane strain, rather than mixed mode, conditions dominated the test, but also "adjusts" $\boldsymbol{K}_{\mathbf{1}}$ 's to be $\boldsymbol{K}_{\boldsymbol{Q}}$ 's if the plane strain thickness were to be decreased. Secondly, it adds a physical constraint as an input variable to the virtual experiments, thereby more closely resembling the actual experiment. An additional practical and important consequence in the prediction of an appropriate thickness that would result in a test dominated by plane strain, and hence $\boldsymbol{K}_{\mathbf{1} C}$, is the possibility to predict, and hence design, a $\boldsymbol{K}_{Q}(\boldsymbol{t})$ where the stress state at the crack tip is positively (and predictably) influenced by the triaxiality at the free surface, rather than an overly conservative $\boldsymbol{K}_{\mathbf{1} \boldsymbol{C}}$ for thin specimens, resulting in smaller (thinner) components and a total weight savings.

The research described in this paper has three principal aims. The first is the development of the necessary databases to relate composition/microstructure and fracture toughness in $\alpha / \beta$-processed Ti-6Al-4V. The second is the development of rules-based models to predict interpolatively (i.e., within the database) the fracture toughness in these alloys. The third is the determination, validation, and interpretation of the functional dependencies derived from the models. It is necessary to develop several types of models to accomplish these tasks and permit the development of a mechanism-based understanding of fracture in $\alpha / \beta$-processed Ti-6Al-4V.

\section{EXPERIMENTAL PROCEDURES}

As described in previous work on the prediction of tensile properties, ${ }^{[9]}$ this research includes an intentional variation of not only microstructural features but also composition. A total of nine different titanium alloys (based around the nominal composition of Ti-6Al-4V) were produced with intentional variations in the relative amounts of the individual elemental species, including the impurity $\mathrm{O}$ and $\mathrm{Fe}$ contents. The variations in alloy compositions, as measured by TIMET's Henderson Technical Laboratory, Henderson, NV using inductively coupled plasma (ICP) spectroscopy, are Ti- $x \mathrm{Al}-y \mathrm{~V}$ $(4.76<x<6.55 ; 3.30<y<4.45)$ with controlled variations in the impurities $\mathrm{O}$ and $\mathrm{Fe}(0.07<$ wt pct $\mathrm{O}<0.20 ; 0.11<$ wt pct $\mathrm{Fe}<0.41)^{*}$. As was the case for

*For reference, AMS specification \#4920 is $5.5<$ wt pct $\mathrm{Al}<6.75$; $3.5<$ wt pet $\mathrm{V}<4.5$; wt pet $\mathrm{O}_{(\max )}=0.20$; and wt pet $\mathrm{Fe}_{(\max )}=0.30$. The $\mathrm{Al}$ content ranged below that of the nominal composition, while the vanadium exhibited a slightly extended range. The maximum Al level was designed to avoid short-range ordering of the hcp phase. Oxygen was designed to exhibit a slightly extended range to cover both extra-low interstitial (ELI) and non-ELI grades. Iron, a known strengthener, was also designed to have an extended range.

the tensile properties, ${ }^{[20]}$ a total of 54 fracture toughness specimens were prepared, corresponding to nine alloy compositions with six different thermomechanical processing histories. These specimens were extracted from the forged billets in a manner designed to minimize differences in plastic deformation strains, and thus minimize differences in texture. For each fracture toughness sample, there was a corresponding tensile sample, as documented in the previous work, with nominally the same microstructure.

The geometry of each fracture toughness specimen was that of a compact tension specimen with a fatigue pre-crack. These specimens were tested at room temperature according to ASTM E399 specifications. The thickness $(\boldsymbol{B})$ was $25.4 \mathrm{~mm}$, and the $\boldsymbol{W}$ and $\boldsymbol{a}$ values were $\sim 51$ and 20.5 to $25 \mathrm{~mm}$, respectively. These geometries were selected based on the estimation that the tests would yield values that would qualify for $\boldsymbol{K}_{\mathbf{1} C}$ measurements, a test that was specified by the specific funding program. Further, the testing was conducted by a certified testing laboratory. Even still, only 23 tests resulted in valid $\boldsymbol{K}_{\mathbf{1} C}$ measurements. The other tests have been reported as $\boldsymbol{K}_{\boldsymbol{Q}}$. It should be noted that of the 24 samples with the highest oxygen contents, all but one were reported as $\boldsymbol{K}_{\mathbf{1} C}$, and consistently had lower fracture toughness values. This is not surprising given the fact that these samples also invariably have higher yield strengths. However, given the variation in type of toughness reported, the models that will be developed in this paper will be for the experimentally less rigorous $\boldsymbol{K}_{\boldsymbol{Q}}$. It is expected that the normalization of the models with the inclusion of the plane strain thickness might give a $\boldsymbol{K}_{\boldsymbol{Q}}$ that is a reasonable approximation of $\boldsymbol{K}_{\mathbf{1}}$.

\section{NEURAL NETWORK DEVELOPMENT}

As noted previously, it is necessary to develop several types of Bayesian Neural Network (BNN) models with sufficient accuracy such that they may be used to develop a mechanism-based understanding of fracture in $\alpha / \beta$ processed Ti-6Al-4V. These are shown schematically in Figures 4(a) through (c) where the inset double triangle captures the interconnected nature of the 
continuum material variables, such as the yield stress and stress state at the crack tip, and their dependencies upon microstructure and composition which may influence the fracture toughness directly. Notably, the extrinsic toughening mechanisms (see Figure 1) are neglected as they can likely be related to individual microstructural features and therefore contribute as identified micromechanisms. The models that will be developed include a baseline model which only includes the continuum variables thereby approximating the legacy understanding (see Figure 4(a)), a model which only includes potential micromechanistic details (see Figure 4(b)), and a model which includes both the continuum and micromechanistic details, including microstructure and composition (see Figure 4(c)). These models, their quality, and their functional dependencies will be shown and the results discussed.

The development of a high-fidelity neural network critically depends upon the careful establishment of a robust, high-fidelity database relating input variables (e.g., composition, microstructure) to an output variable (e.g., fracture toughness). The first aspect of developing a neural network model is the rigorous development of such a database. Therefore, following fracture toughness, an undeformed section of the fracture toughness blocks was excised and prepared for metallographic analysis using conventional specimen preparation techniques. Following preparation, the samples were characterized in an identical manner described elsewhere, ${ }^{[20]}$ and making use of a FEI/Philips Sirion scanning electron microscope (SEM) operating in backscattered electron (BSE) mode at $15 \mathrm{kV}$. The backscattered electron micrographs were collected in a random and unbiased fashion, and quantified using stereological techniques developed for these types of $\alpha / \beta$-processed Ti-6Al-4V microstructures. ${ }^{[23]}$ The features that were quantified are the size and volume fraction of the equiaxed alpha particles (equiaxed- $\alpha$ size $(\mu \mathrm{m})$ and $\left.F_{V}^{\text {equiaxed-alpha }}\right)$, the volume fraction of total alpha ( $F_{V}^{\text {total-alpha }}$, and the thickness of the alpha laths in the transformed $\beta$ regions $(\alpha$-lath width, $\mu \mathrm{m}) .^{[23]}$ These microstructural features, and the corresponding
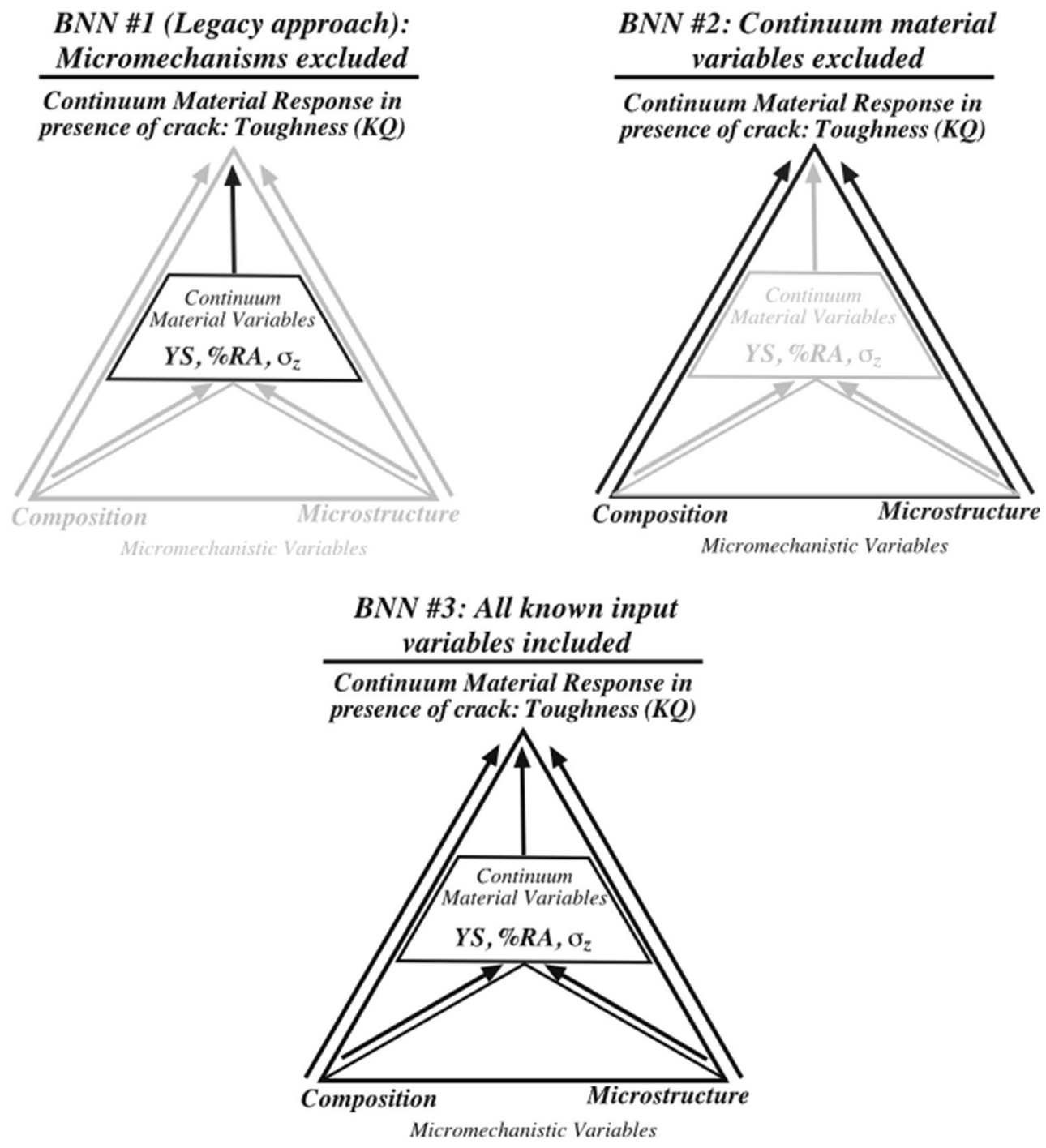

Fig. 4-Schematic diagram to illustrate the various sets of input data used to generate the neural network models. 
compositions, were first used as inputs to predict their yield strengths, based on the well-developed and validated model developed in previous work. ${ }^{[20]}$ The results were then compared to tensile specimens that had nominally the same thermomechanical processing history, and therefore, nominally, the same microstructure and properties. The blind predictions based upon the model developed in Reference 20 were within \pm 2.5 pct of the data obtained from the corresponding tensile tests, further validating the results of the previous work. Thus, while the corresponding experimentally measured yield strength and reduction in area from the tensile tests were used as inputs to the models developed in this paper to reduce the possibility of the propagation of a large uncertainty between yield strength and toughness models, there is considerable confidence that the influence of composition and microstructure on toughness in the various models developed is accurate.

A database was developed containing experimentally determined data, including (i) alloy composition, specifically, Al, V, O, and Fe; (ii) materials microstructure, including phase fractions and average sizes of key microstructural features; (iii) the yield strength and reduction in area obtained from uniaxial tensile tests; (iv) an assessment of the degree to which plane strain conditions were satisfied by including a plane strain thickness factor; and (v) the fracture toughness. The plane strain thickness factor is calculated from an average of 5 measurements across the fracture surface, as illustrated in Figure 5. While it is acknowledged that the true plane strain thickness will likely be greater than that estimated (closer to the crack tip prior to ductile rupture), this approach accounts for the curvature which is set in part by the true plane strain thickness, and hence the variability of the plane strain thickness is captured but in a determinable fashion.

This database was used to train neural networks to predict interpolatively the fracture toughness of the alloy Ti-6Al-4V, based on varying combinations of microstructure, composition, yield strength, reduction in area, and plane strain thickness. Fourteen combinations of inputs were used to generate the models. Four sets of inputs included the combinations of the three continuum contributions mentioned above (YS, RA, $\left.t_{\text {plane strain }}\right)$, and 8 sets included microstructure and composition. In this way, it is possible to explore separately the influence of microstructure/composition on fracture toughness through its modification of continuum variables (e.g., yield strength) and microstructure/composition on fracture toughness through extrinsic means. In addition, two sets of inputs were used to develop models which may be used to predict the plane strain thickness. One of these two sets of inputs directly included composition and microstructure, while the other included yield strength and reduction in area.

The artificial neural networks were trained following the Bayesian scheme described by Mackay. ${ }^{[21,22]}$ Typically, when the neural network is being trained and tested, one particular model emerges as a superior model, based on the minimization of the residual error using a mean square error (MSE) approach with respect to the experimental values. When this superior model is further interrogated for the determination of the functional dependencies, by carrying out virtual experiments (see below), each of the dependencies will represent a physical reality that is easily interpreted. For example, the influence of oxygen on the yield strength in $\sigma+\beta$ processed Ti-6-4 emerges as the most potent strengthening mechanism, and is in keeping with what is expected given the legacy data. However, as described previously, the absence of legacy information relating microstructural features with fracture toughness, in addition to the complex nature of fracture in general, creates a situation where it is appropriate to consider more than the single model with the lowest MSE. Therefore, for each of the 14 sets of inputs (12 for the interpolative prediction of fracture toughness and 2 for plane strain thickness), a total of 288 models were developed with different model architectures (i.e., different seed values, number of nodes, and sigma widths). Of these 288 models, at a minimum, the three best models with the lowest MSE values were subsequently investigated.

\section{USE OF THE NEURAL NETWORK MODELS}

Once developed, the neural network can be used in one of two ways. Firstly, neural networks can be used to develop a predictive tool, where the properties of a testing (not training) dataset are predicted. The test dataset represents a fraction of the 54 samples that were not used for the training; in this case, 40 samples were used to train the model and 14 to test the model. Secondly, the neural network model can be used to conduct virtual experiments, as described above. These are control experiments where the values of given microstructural features can be set to, or held at, given values. In this way, it is possible to obtain information from virtual experiments where such control of individual microstructural features may not be possible experimentally. One such virtual experiment is the determination of the functional dependence of a given property on the selected input variable. Thus, the functional dependencies are determined by setting all but one variable at a fixed average value and allowing that single variable to vary while observing the change in the predicted fracture toughness. It is acknowledged that it would not be possible to generate some of the combinations of inputs physically (in the laboratory), but, nevertheless, it is the trend exhibited by these virtual experiments that is of most use regarding the provision of an insight into the functional dependency of the property on the given variable.

\section{RESULTS AND DISCUSSION}

It is useful to first provide an example illustrating how the chemistry, microstructure, and tensile properties all contribute during fracture propagation (and thus are captured in a measure of fracture toughness) in off-setting ways. Consider first the three distinctly difference microstructures shown in Figures 6(a) through (c). 


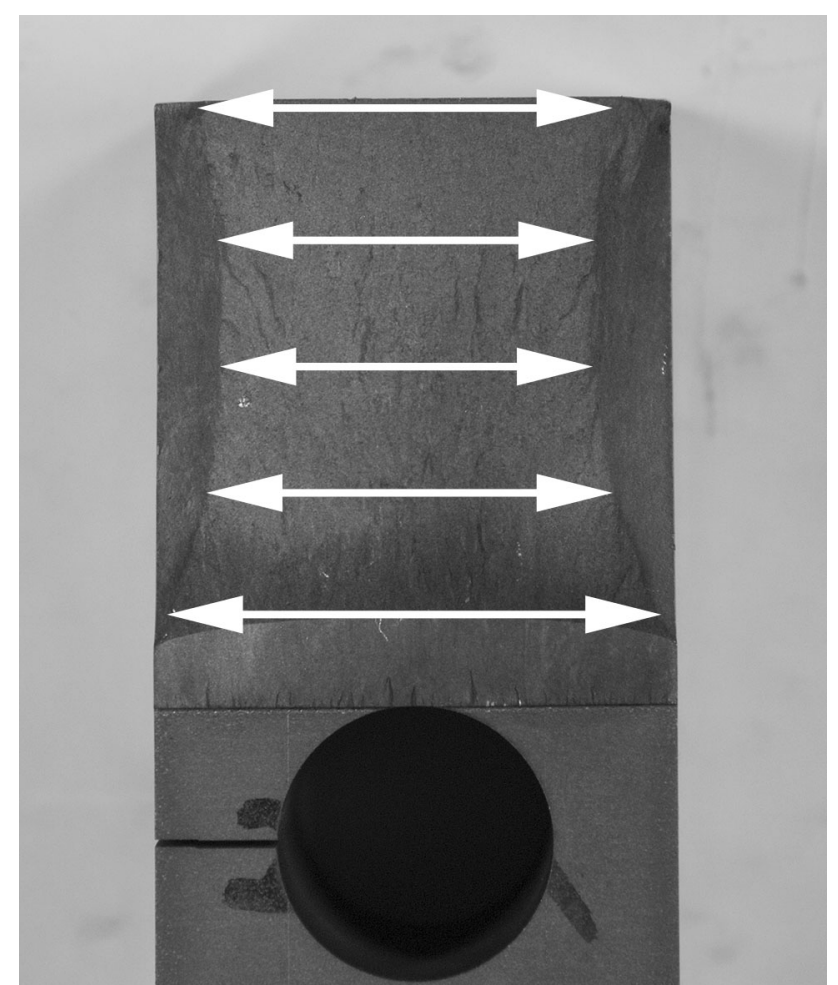

Fig. 5-The way in which plane strain thickness measurements have been made.

These three microstructures have been quantified, and the stereologically determined metrics presented in Table I. Also included in Table I are the chemistries and yield strengths for each specimen. It is clear that the microstructure, chemistry, and yield strength are distinctly different. Yet, despite these differences, these three samples have a statistically identical fracture toughness (see Table I, $\boldsymbol{K}_{\boldsymbol{Q}} \sim 83 \mathrm{MPa} \sqrt{\mathrm{m}}$ ). This is not to suggest that titanium alloys all have a single value of fracture toughness. Indeed, to the contrary, as shown in Figure 2, the fracture toughness can range from $\sim 40$ to $110 \mathrm{MPa} \sqrt{\mathrm{m}}$, depending upon composition and microstructure. Even for the Ti-6Al-4V database developed here, the toughness ranges from $\sim 50$ to $\sim 100$ $\mathrm{MPa} \sqrt{\mathrm{m}}$. Rather, Figures 6(a) through (c) and the corresponding Table I illustrate the problems experimentalists have faced when attempting to decouple the composition, microstructure, and continuum variables when interpreting the measured toughness.

The microstructures shown in Figures 6(a) through (c) have resulted in samples whose experimentally measured yield strengths vary by more than 12 pct (725 to $832 \mathrm{MPa}$ ). From this result, it is reasonable to conclude that yield stress alone cannot be used as a single input variable to a model which predicts fracture toughness. Rather, microstructural features also play a role, to be established through virtual experiments (see below). Thus, in addition to modifying the local tensile properties and hence the stress state at the crack tip, microstructure may also affect extrinsic phenomena or micromechanisms such as the crack path modification, shown schematically in Figure 1.
The results which follow demonstrate that, for complex problems such as fracture toughness, multiple neural network architectures can be developed and the resulting models probed to separate interdependent terms. For fracture toughness, it will be shown that it is possible to isolate the micromechanistic and continuum contributions. For the purposes of this work, the continuum variables are yield strength, ductility (reduction in area), and a factor that scales with plane strain thickness. Indirectly, these continuum variables will allow for the investigation of the effect of crack tip fields (e.g., plane strain $v s$. mixed mode $v s$. plane stress conditions) on the reported value of $\boldsymbol{K}$ (i.e., $\boldsymbol{K}_{1 C}$ vs. $\boldsymbol{K}_{\boldsymbol{Q}}$ ).

\section{A. Development and Interpretation of Models for the Prediction of Toughness}

A variety of methods exist to evaluate the accuracy of a model. The first involves an analysis of both the deviation of a prediction from an experimentally determined value as well as the predicted errors of the models. The second involves an analysis of the mean square of the errors (MSEs). The latter will often help separate seemingly similar models, and is often used to pick "the optimum" model. For the former, the average $(\bar{\delta})$ and maximum deviations $\left(\delta_{\max }\right)$ and errors $(E)$ are reported. For the latter (e.g., the interpretation of model accuracy based upon MSEs), the three models with the lowest MSE for each of the 12 model types have been averaged and are reported.

Figures 7 (a through d) show the predicted $v s$. experimental toughness for four representative models, while Table II summarizes the numerical analysis of all 12 models, with the four shown in Figure 7 highlighted for ease of comparison. Figure 7(a) shows the best model that excludes direct input of microstructure and composition, specifically the model with only the three continuum variables included. This model has a maximum deviation from experiment of 19.5 pet and an average deviation from experiment of 3.5 pct. Although this is the best "baseline" model which mimics the legacy understanding of toughness in Ti-based alloys (i.e., it only includes continuum effects and does not directly include either microstructure or composition), it is a considerably poorer model than those that include such inputs. It is illustrative to note that this scatter is analogous to the data presented in Figure 2, reflecting the legacy approach. Figures 7(b) through (d) include either microstructure and composition without any direct inclusion of continuum inputs (see Figure 7(b)) or composition and microstructure in addition to continuum inputs, such as yield strength (Figure 7(c)) or yield strength, plane strain thickness, and reduction in area (Figure $7(\mathrm{~d})$ ). These three models, in addition to having superior MSEs (15.6, 10.2, 10.6, respectively), have significantly lower average and maximum deviations from prediction $\left(\delta_{\mathrm{avg}}: 1.8,1.1,0.9 ; \delta_{\max }: 7.9,5.6\right.$, 5.8) than the model shown in Figure 7(a).

The results of these numerical analyses demonstrate two significant factors when considering the development of data-informatics tools for the prediction of the 
toughness of a material, including artificial neural networks. Firstly, in order to reduce the uncertainty and improve the quality of the models, it is necessary to include details regarding microstructural and compositional inputs in addition to the inclusion of continuum variables. Excluding microstructure and composition (i.e., focusing only on the influence of yield strength) will lead to significant scatter in the values of the predicted yield strengths when correlated with toughness (see Figure 7(a)). This model indicates that such scatter is rather significant, and the raw data obtained for this research indicate that such scatter exceeds 20 pct. Secondly, of the models that do include compositional and microstructural inputs, the models that also include yield strength directly are far superior to those which do not. When considering a further analysis of the mean square errors (average ranging from 10.2 to 15.6), it is apparent that the mean square of the errors are all less than 11.52 for models where yield strength is included, whereas those that did not directly include yield strength were all greater than 12.89. Similarly, an analysis of the maximum deviation $\left(\delta_{\max }\right)$ shows that the four models which include yield strength in addition to the composition/microstructure have an average maximum deviation of 6.25 pct, while the four models which exclude yield strength but include composition/microstructure have an average maximum deviation of 8.65 pct, also indicating a poorer quality model. These observations reinforce the importance of considering the continuum effect of yield strength on establishing the stress state at the crack tip, independent of the effect, if any, that specific local microstructural features may have on the micromechanisms of toughness. However, it is equally clear that the inclusion of microstructure and composition as inputs leads to improvements in the models which may then be interrogated to help determine possible mechanisms.

It is important to identify the effect that the input variables have on toughness. However, as previously described, this is particularly challenging for fracture toughness since a given effect of microstructure on a property such as yield strength (i.e., an important continuum variable) does not necessarily cause a rigorously corresponding effect (either $+/-/$ neutral) on toughness nor its microscale phenomena, such as crack propagation or possible extrinsic toughening/weakening mechanisms. Therefore, rather than only focusing on the best models (i.e., Model \#6 or \#12), a series of virtual experiments to explore functional dependencies has been developed for each of these models in order to compare them and thus explore the effect of the microstructural and compositional variables on toughness, both in conjunction with and isolated from the continuum variables. As will be seen, this novel simultaneous application of different neural network architectures to such a complex problem has resulted in some newly developed understandings of the contributions of microstructure to fracture toughness in $\sigma+\beta$ processed Ti-based alloys.

\section{B. Virtual Experiments Using the Neural Networks}

\section{Effect of yield strength on toughness}

Figures 8(a) through (c) show the functional dependence of yield strength on $K_{Q}$ for three models**. For

\footnotetext{
**The numerical component of the model designations in the figure captions for Figures 8, 9, 10, 11, 12, 13 corresponds to the neural network architecture itself. The format is value of the seed-number of hidden nodes-initial Gaussian width. For example, "500-3-1" corresponds to a value of the seed number (500) for an architecture with 3 hidden nodes, and an initial Gaussian width of 1 , as described in detail elsewhere. ${ }^{[26]}$
}

each functional dependence, there is a significant decrease in $K_{Q}$ with increasing yield strength for all models which include as input yield strength as well as compositional and microstructural variables. Given the legacy data shown in Figure 2, such a decrease is expected. Additionally, Figures 8(a) through (c) include trends calculated from the three best model architectures. These trends show clearly that the functional dependencies obtained for each model architecture are quite similar, indicating that the models have interrogated the databases, identified a strong dependence, and established a nearly identical weighting function. This would occur when there are strong correlations to be made among the data. Thus, an advantage of using three model architectures emerges, as it is possible to further assess the overall accuracy of a particular functional dependency. As will be shown below, the functional dependencies for the "best" model architectures often exhibit such remarkably consistent trends, indicating clearly the degree of importance and influence of a particular input variable. However, the authors also observed some cases where the forms of the functional dependencies are rather different for the "best" model architectures. Such differences were often observed in conjunction with either an insignificant dependence (i.e., minimal influence of a feature upon a property) or an anomalously complex dependency, including several that appear to exhibit second or higher order natures, indicating a likely over-fitting of the model to a particular variable.

Figure 8(c) includes an estimation of plane strain thickness. This model, when compared with models that exclude the plane strain thickness (see Figures 8(a) through (b)), shows that the yield strength has less of an influence on the fracture toughness. Indeed, the slope decreases by $\sim 40$ pct when plane strain thickness is included. This result is reasonable, and is readily explained by the fact that the database consists of both $\boldsymbol{K}_{\boldsymbol{Q}}$ and $\boldsymbol{K}_{\mathbf{1} \boldsymbol{C}}$ data. Invariably, the $\boldsymbol{K}_{\mathbf{1} \boldsymbol{C}}$ will be lower than $\boldsymbol{K}_{\boldsymbol{Q}}$. Recall that yield strength influences the stress state $\left(\sigma_{z}\right)$ at the crack tip, and hence, for a given specimen thickness, whether plane strain conditions are valid. This $\sim 40$ pct decrease in the magnitude of the influence of yield strength on toughness, when considered with the effect of plane strain thickness on toughness discussed in 



Fig. 6-Backscattered electron micrographs taken from three different samples which exhibit nominally the same $\mathrm{K}_{\mathrm{Q}}$.

Table I. Composition, Microstructure, and Tensile Properties of Figs. 6(a Through c)

\begin{tabular}{lcccccccccc}
\hline Sample ID & $\mathrm{Al}$ & $\mathrm{V}$ & $\mathrm{Fe}$ & $\mathrm{O}$ & $\begin{array}{c}F_{V} \text { Total } \\
\text { Alpha }\end{array}$ & $\begin{array}{c}\text { Equiaxed } \alpha \\
\text { Size }(\mu \mathrm{m})\end{array}$ & $F_{V}$ Equiaxed $\alpha$ & Thickness $(\mu \mathrm{m})$ & Strength $(\mathrm{MPa})$ & $K_{Q}$ \\
\hline $85-1$ & 4.76 & 4.27 & 0.39 & 0.07 & 87.3 & 5.57 & 68.9 & 0.316 & 725 \\
$100-3$ & 5.64 & 3.83 & 0.25 & 0.14 & 92.2 & 5.93 & 44.5 & 0.350 & 83.6 \\
$109-1$ & 6.51 & 4.29 & 0.11 & 0.08 & 89.9 & 6.54 & 70.4 & 0.361 & 832 & 790 \\
\hline
\end{tabular}

the subsequent section, suggests that the neural networks are acting to scale experimentally measured $\boldsymbol{K}_{\boldsymbol{Q}}$ 's to an estimate of a $\boldsymbol{K}_{\mathbf{1} \boldsymbol{C}}$ if the thickness were to increase, as well as scale experimentally measured $\boldsymbol{K}_{\mathbf{1}}$ 's to an estimate of $\boldsymbol{K}_{\boldsymbol{Q}}$ if thickness were decreased.

As is evident, there is still a significant effect that yield strength has on toughness, independent of the effect of stress state at the crack tip. The model (see Figure 8(b)) that includes the reduction in area does not decrease the dependency of toughness on yield strength. Thus, yield strength must influence toughness in ways beyond a simple stress-strain argument. While the exact cause of this inverse relationship is not known for $\alpha / \beta$-processed Ti-6Al-4V, a two-phase, elastic-plastic, strain-hardening material, the following phenomena are possible. Firstly, the crack tip opening displacement is likely to be less for samples with higher yield strengths. A smaller crack tip opening displacement would result in a decrease in the crack tip radii, resulting in a high stress distribution over a greater distance from the crack tip. This could result in a greater volume of material experiencing a higher stress, which, if above a critical stress (e.g., a critical resolved shear stress for dislocation motion), would make possible the accumulation of damage in front of the crack tip, including the formation of new pores or cracks, and ultimately decreasing the fracture toughness. Secondly, though less likely, a microstructural feature or compositional variable may exist that does not influence the uniaxial tensile properties (yield strength, ductility) but might decrease the material's damage tolerance. Lastly, reflect that ligament bridging is essentially a series of tensile tests. If ligament bridging does occur, which is highly probable for a two-phase ductile material, the yield strength of the material would be directly proportional to the crack tip radius and, thus, to the crack propagation. While multiple mechanisms might be operating, the determination of which is the operative mechanism is not trivial and has not been determined in this work.

\section{Effect of plane strain thickness on toughness}

While the plane strain thickness does modify $\boldsymbol{K}_{\boldsymbol{Q}}$, the magnitude of this contribution is often not clear (see Figure 3). A classical estimation shown by Irwin of $\boldsymbol{K}_{\boldsymbol{Q}}$ incorporating $\delta_{1 \mathrm{C}}$ is given as

$$
K_{Q}=K_{C}=K_{1 C}\left(1+1.4\left(\beta_{1 C}\right)^{2}\right),
$$

where

$$
\beta_{1 C}=\frac{1}{t}\left(\frac{K_{1 C}}{\sigma_{y s}}\right)^{2} .
$$

These equations give an empirical relation based upon a true plastic zone size calculation (and hence upon a rigorous determination of both $\boldsymbol{K}_{1 C}$ and yield strength) and would result in a $\boldsymbol{K}_{\boldsymbol{Q}}$ which does not approximate, indeed, far overestimates the $\boldsymbol{K}_{\boldsymbol{Q}}$ 's obtained in this work 
for this alloy for the thickness of our specimens. Thus, it is still a challenge to determine the influence of thickness upon the measured toughness $(\boldsymbol{K})$ and whether that toughness represents a valid $\boldsymbol{K}_{\mathbf{1} C}$ or plane stress-dominated $\boldsymbol{K}_{\boldsymbol{Q}}$. It is equally challenging to determine the appropriate experimental conditions based upon classical fracture mechanics, as the approximation for $\boldsymbol{B}$ (sample thickness) $\left(\boldsymbol{B} \geq 2.5\left(\boldsymbol{K}_{\mathbf{1} \boldsymbol{C}} / \sigma_{\mathrm{ys}}\right)^{2}\right)$ represents a minimum thickness before plane strain behavior may occur, and not an absolute prediction that it will occur. The variation in whether plane strain behavior occurs therefore must be related to intrinsic material variability, including the effects of both microstructure and composition. Therefore, it is notable that by including both $\boldsymbol{K}_{\boldsymbol{Q}}$ 's and $\boldsymbol{K}_{\mathbf{1}}$ 's in this model, and by including a factor to incorporate the variation in plane strain thickness, the possibility of estimating the effect of thickness on the toughness variability that exists between $\boldsymbol{K}_{\mathbf{1} \boldsymbol{C}}$ and $\boldsymbol{K}_{\boldsymbol{Q}}$ has been determined.

Consider Figure 9 which presents the influence of plane strain thickness on $\boldsymbol{K}_{\boldsymbol{Q}}$ when yield strength is included in the models, and is held at a fixed "average" value, i.e., when undertaking a virtual experiment. In this figure, the samples with a smaller plane strain thickness (i.e., effectively those with $\boldsymbol{K}_{\boldsymbol{Q}}$ values) exhibit predicted toughness values up to $\sim 9 \mathrm{MPa} \sqrt{\mathrm{m}}$ greater than their $\boldsymbol{K}_{\mathbf{1}}$ microstructural/compositional analogs. As was done with the effect of yield strength, the functional dependencies and their predicted errors obtained using the three best model architectures are shown to illustrate the similarity among them. Indeed, the results show a negligible difference between the three best model architectures indicating a very clear effect of variable on property. The influence of plane strain thickness on the toughness suggests that the magnitude of the difference between a true $\boldsymbol{K}_{1 C}$ and a measured $\boldsymbol{K}_{Q}$ can be up to at least 10 pct of the value for $\alpha / \beta$-processed Ti-6Al-4V. The interplay between yield strength, plastic zone size, sample thickness, and $\boldsymbol{K}_{Q} / \boldsymbol{K}_{\mathbf{1} C}$ still represents a complex problem - but the current research allows for a first approximation of their interdependencies- experimentally clarifying the order of magnitude difference that is often schematically illustrated in representations such as Figure 3.

\section{Determining the functional dependence of} microstructure and composition on fracture toughness

Three of the microstructural features that affect fracture toughness are the volume fraction equiaxed alpha, the size of the equiaxed alpha, and the thickness of the alpha laths in the transformed beta regions. For a model that includes only composition and microstructure, the three functional dependencies are shown in Figures 10(a) through (c). Clearly, the volume fraction equiaxed alpha significantly affects the toughness $(\sim 14 \mathrm{MPa} \sqrt{\mathrm{m}})$, with lesser effects attributed to the size of the equiaxed alpha $(\sim$ $10 \mathrm{MPa} \sqrt{\mathrm{m}})$ and the thickness of the alpha laths $(\sim 7$ $\mathrm{MPa} \sqrt{\mathrm{m}})$. However, when the continuum variables are included as separate inputs, as in the model leading to Figure 8(d), the functional dependencies (see Figures 11(a) through (c)) allow for differentiation regarding which features contribute to the continuum and which features are directly related with micromechanisms. Consider the significant reduction in the degree to which toughness is dependent upon volume fraction equiaxed alpha when continuum variables are included. Thus, in a model that includes yield strength, the contribution of volume fraction equiaxed alpha becomes negligible $(\sim 0$ to $1 \mathrm{MPa} \sqrt{\mathrm{m}})$. This strongly suggests that the volume fraction equiaxed alpha only affects toughness by modifying the macroscopic yield strength of the material. When the results of the investigation into the influence of composition and microstructure on tensile properties ${ }^{[9]}$ are considered, it is clear that this is the microstructural variable that has the largest influence on the tensile properties over the range that can be affected. Similarly, consider the much smaller reduction in the degree to which toughness is dependent upon the size of the equiaxed alpha when yield strength is included. In the model that includes yield strength, the contribution of equiaxed alpha size has the greatest effect $(\sim 7 \mathrm{MPa} \sqrt{\mathrm{m}})$. Lastly, consider the influence of the effect of the thickness of the alpha laths, both with and without yield strength in the model. Inclusion of continuum variables yields a similar result as that noted for the influence of volume fraction equiaxed alpha. Thus, it appears that this microstructural variable does not directly influence fracture toughness. From consideration of Figure 11, it appears that the equiaxed alpha size (or another microstructural feature directly linked to the size of the equiaxed alpha) has, at the very least, as significant an influence on the micromechanisms of fracture as it does on the continuum variables.

In a similar fashion, the functional dependencies of the compositional variables have been determined, and their influence upon the fracture toughness explored. The functional dependencies obtained for a model including microstructure and composition but not yield strength are shown in Figures 12(a) through (d), while the functional dependencies obtained for a model including microstructure, composition, and yield strength are shown in Figures 13(a) through (d). Two primary observations can be made for these two figures. The first observation is the manner in which both $\alpha$-stabilizers (Al and $\mathrm{O}$ ) influence the toughness. These functional dependencies exhibit relatively clear trends for the model without yield strength included (Figures 12(a) and (b)). The trends are fairly consistent for models developed with different model architectures. This is expected, given the strong positive influence of $\mathrm{Al}$ and $\mathrm{O}$ on yield strength, as well as the strong negative influence of YS on toughness. However, the implications of the functional dependencies are less clear for the model that includes yield strength. Indeed, the two best combinations of model architectures produce significantly different trends. Additionally, the error bars are significantly greater, and the shape of one of the $\mathrm{O}$ functional dependencies is rather complex. It should be noted that the raw data, which have already produced exceptionally good dependencies of the composition on the tensile properties, are taken from within the same billets as these toughness specimens. Therefore, the accuracy of the input compositional variables appears not to be in doubt. Thus, it is reasonable to consider that 
Model - Continuum Variables

(yield strength, reduction in area, plane strain thickness)

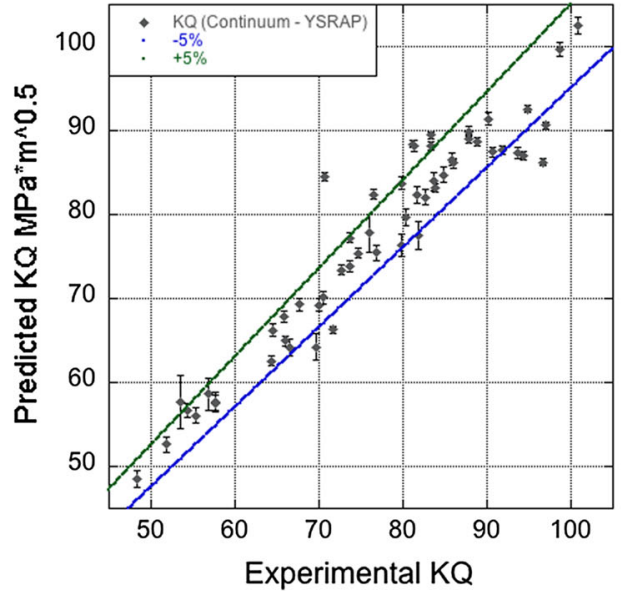

(a)

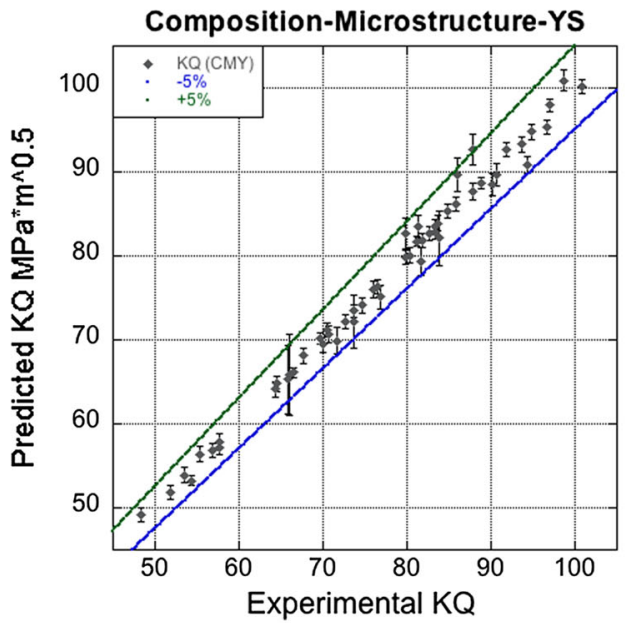

(c)

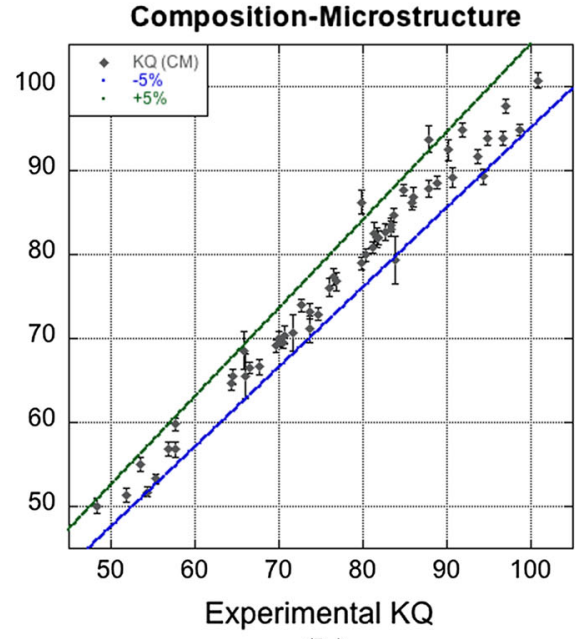

(b)

Composition-Microstructure-Plane Strain-YS-RA

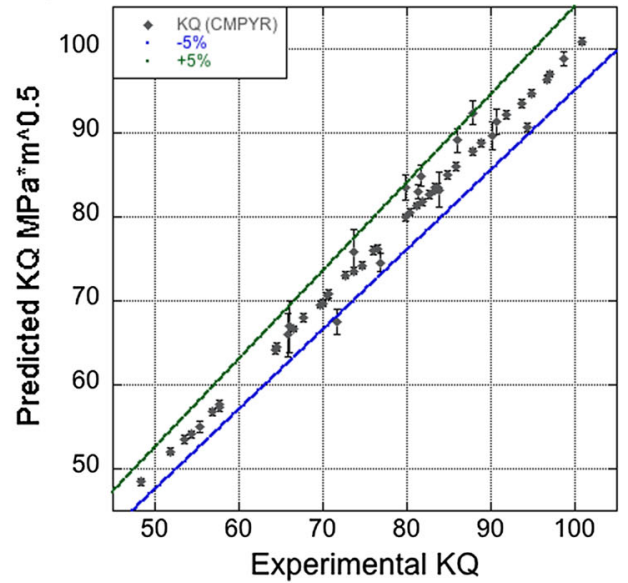

(d)

Fig. 7-(a) The best predicted vs. experimental plot of $K_{Q}$ that excludes composition and microstructure and $(b$ through $d)$ the predicted $v s$. experimental plots of $K_{Q}$ for three example models which include composition and microstructure.

Table II. Numerical Analysis of Model Quality. Corresponding Components of Fig. 7 are Labeled

\begin{tabular}{|c|c|c|c|c|c|c|c|c|c|}
\hline \multirow[b]{2}{*}{ Model } & \multicolumn{4}{|c|}{ Inputs } & \multirow[b]{2}{*}{ Avg. MSEs } & \multicolumn{4}{|c|}{ Best Model } \\
\hline & YS & RA & $t_{\text {pl.strain }}$ & Comp/Micro & & $\bar{\delta}$ & $\delta_{\max }$ & $\bar{E}$ & $E_{\max }$ \\
\hline$\# 1$ & $\sqrt{ }$ & $\sqrt{ }$ & & & & 19.1 & 57.3 & 1.5 & 5.6 \\
\hline$\# 2$ & $\sqrt{ }$ & & $\sqrt{ }$ & & & 5.4 & 26.7 & 0.9 & 2.2 \\
\hline \#3 & & $\sqrt{ }$ & $\sqrt{ }$ & & & 6.8 & 28.4 & 0.6 & 1.6 \\
\hline$\# 4$ (7a) & $\sqrt{ }$ & $\sqrt{ }$ & $\sqrt{ }$ & & & 3.5 & 19.5 & 1.2 & 5.4 \\
\hline \#5 (7b) & & & & $\sqrt{ } / \sqrt{ }$ & 15.61 & 1.8 & 7.9 & 1.4 & 4.1 \\
\hline$\# 6$ (7c) & $\sqrt{ }$ & & & $\sqrt{ } / \sqrt{ }$ & 10.20 & 1.1 & 5.6 & 1.6 & 7.3 \\
\hline$\# 7$ & & $\sqrt{ }$ & & $\sqrt{ } / \sqrt{ }$ & 13.78 & 1.4 & 6.9 & 1.7 & 7.2 \\
\hline$\# 8$ & & & $\sqrt{ }$ & $\sqrt{ } / \sqrt{ }$ & 14.40 & 1.6 & 12.3 & 1.5 & 4.3 \\
\hline$\# 9$ & $\sqrt{ }$ & & $\sqrt{ }$ & $\sqrt{ } / \sqrt{ }$ & 11.52 & 1.3 & 7.4 & 1.4 & 4.3 \\
\hline$\# 10$ & & $\sqrt{ }$ & $\sqrt{ }$ & $\sqrt{ } / \sqrt{ }$ & 12.89 & 1.0 & 7.5 & 1.1 & 6.1 \\
\hline \#11 & $\sqrt{ }$ & $\sqrt{ }$ & & $\sqrt{ } / \sqrt{ }$ & 11.16 & 0.9 & 6.2 & 1.8 & 11.4 \\
\hline$\# 12$ (7d) & $\sqrt{ }$ & $\sqrt{ }$ & $\sqrt{ }$ & $\sqrt{ } / \sqrt{ }$ & 10.55 & 0.9 & 5.8 & 1.0 & 4.6 \\
\hline
\end{tabular}




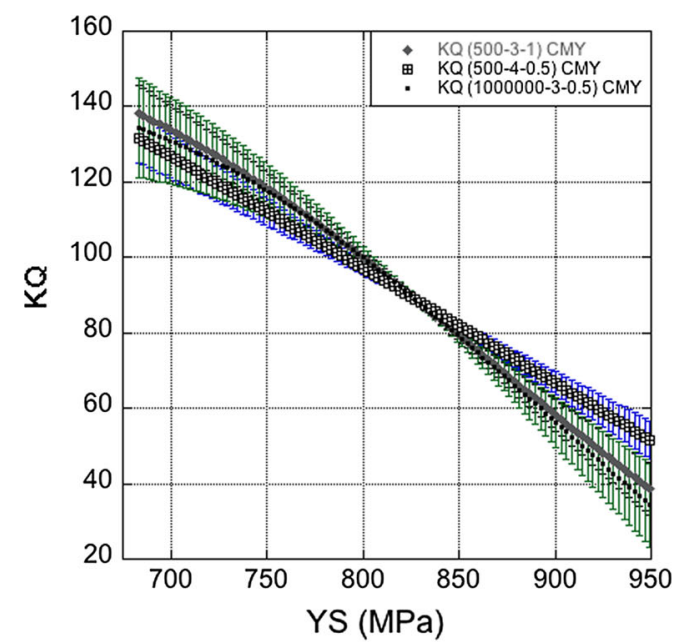

(a)

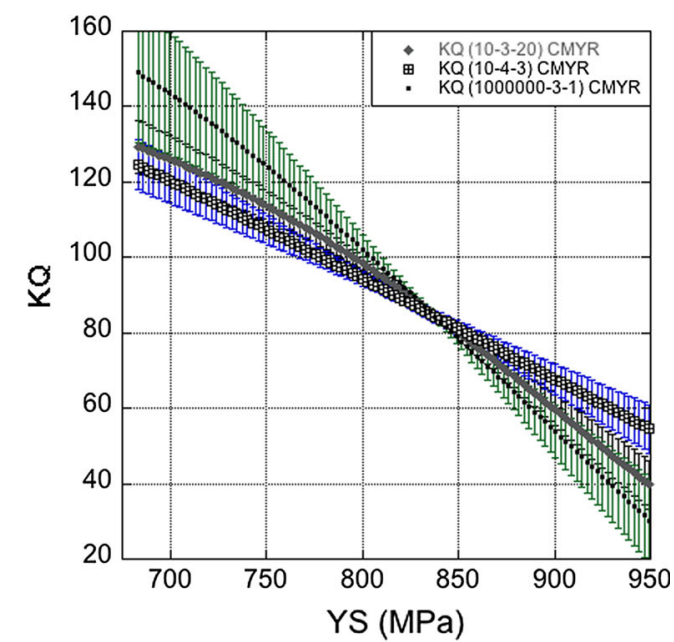

(b)

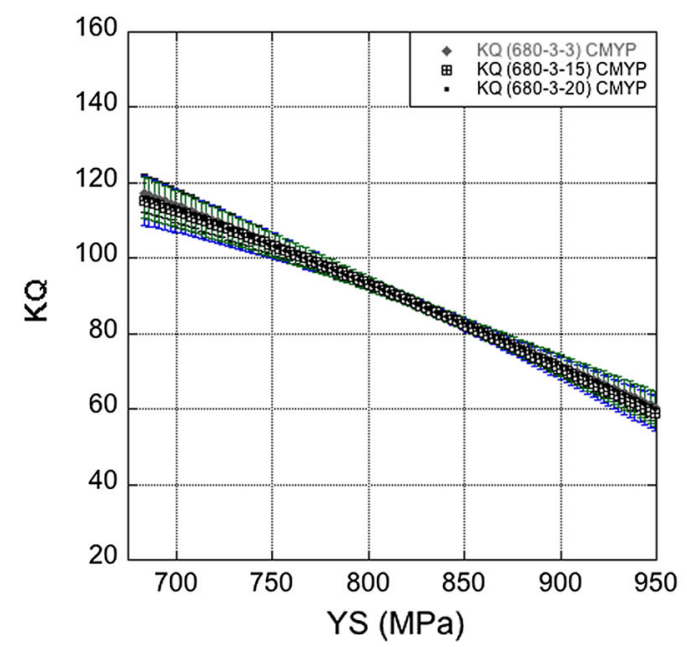

(c)

Fig. 8-Result of virtual experiments: effect of YS on $K_{O}$ as determined by models which include $(a)$ composition-microstructure-yield strength, (b) composition-microstructure-yield strength-reduction in area, $(c)$ composition-microstructure-yield strength-plane strain thickness.

either these trends are reasonable facsimiles of a complex reality or neither $\mathrm{Al}$ or $\mathrm{O}$ directly affect the micromechanisms of fracture in an unambiguous fashion captured by models which include yield strength. The second observation is the manner in which both $\beta$-stabilizers ( $\mathrm{V}$ and $\mathrm{Fe}$ ) influence the toughness. The functional dependencies for each of these species show an increase in toughness for increasing solute content for models excluding yield strength. Additionally, although $\mathrm{V}$ appears to have a neutral effect on toughness once yield strength is included, it does appear that increased $\mathrm{Fe}$ still has a positive influence on toughness. The trends in the composition of Fe are contrary to the previous observations which clearly showed an inverse relationship between strength and toughness for the other seven compositional or microstructural variables. Only Fe resulted in trends that indicate an increase in $\mathrm{Fe}$ results in an increase in both yield strength and toughness.

Both the equiaxed alpha size and the Fe levels may be rationally related to the fracture toughness in the following way ${ }^{\dagger}$. A more detailed explanation may be

${ }^{\dagger}$ The full extent of this research cannot be adequately presented in a single publication. The characterization activities (though published first ${ }^{[24]}$ ) were extensively informed by this modeling effort. Indeed, without the identification of the importance of the size of the equiaxed alpha particle size, the characterization efforts would not have been pursued as carefully.

found elsewhere. ${ }^{[24]}$ The critical microstructural feature is a special twist boundary, whereby adjacent equiaxed alpha particles have parallel (0001) planes (parallel to within a degree or two). A necessary condition is that the interface must also be parallel to the (0001) plane of both adjacent equiaxed alpha particles. When the (0001) plane normal is rotated by up to $30 \mathrm{deg}$, the material has a higher probability of accumulating critical damage in the form of microcracks. These microcracks will "split open" the (0001) interfaces, and terminate when the interface plane deviates. Thus, not all particles with parallel (0001) planes will experience microcracks, only 
those where the interface is also parallel. This particular interface is relatively rare, yet is correlated with a majority of microcracks near the fracture surface. The role of $\mathrm{Fe}$ is less clear, but seems to be related to a very thin layer of beta that separates the adjacent equiaxed alpha particles. As Fe stabilizes the beta phase, the thickness of this thin layer increases, separating the equiaxed alpha particles. One argument may be that a thicker beta region can more easily accommodate strain. Another is that this particular (0001) $30 \mathrm{deg}$ rotation would result in (effectively) a face-centered cubic stacking at the interface, which is an especially high-energy interface and subject to decohesion following Irwin's energy theory. As another phase (beta) is stabilized, the high-energy interface would be eliminated.

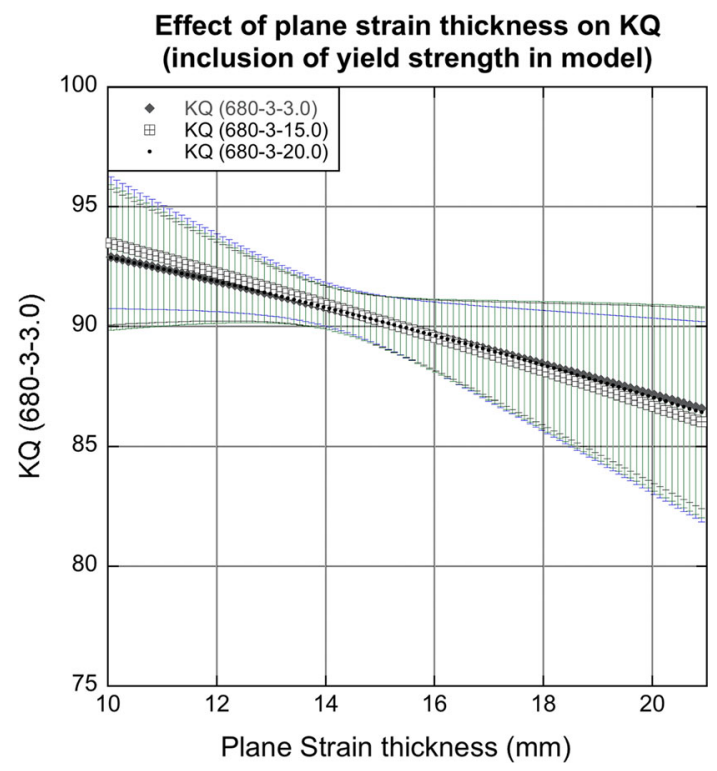

Fig. 9-Result of a virtual experiment: The functional dependence of plane strain thickness on $K_{Q}$ when yield strength is included in model.

\section{Extension of this Approach to Other Problems}

Traditionally, neural network models are applied to problems where the dependency of a determinable output (e.g., a property) on a series of inputs (e.g., microstructure, composition) can be understood by an optimized relationship. Even more complex "committee" neural network models ${ }^{[26]}$ rely upon, at the very least, the existence of a dependency of an output on an input. This work has demonstrated that there exist certain problems that are best studied by comparing the functional dependencies of models where the input variables change. From the perspective of neural networks, effectively this says that there exist certain problems for which measurable inputs can be exist in not only the input layer (e.g., composition, microstructure), but also the hidden layer (e.g., yield strength). From the perspective of materials science problems, this approach can be extended to any problem where one measurable continuum output variable influences another measurable property. It is demonstrated here for a "weak-link"-driven problem, and is likely extendable to other weak-link-driven phenomena, including low cycle fatigue or any other complex processes where $c=f(a, b)$, and $d=f(a, b, c)$.

\section{CONCLUSIONS}

Multiple architectures have been adopted to establish Bayesian Neural Network models. The models have been based upon a well-developed database with systematic variations in composition, microstructure, and tensile properties. The models based upon different input architectures have been assessed to determine how composition and microstructure influence the fracture toughness of $\sigma+\beta$ processed Ti-6Al-4V. From these models, it is possible to differentiate the continuum and mechanistic aspects of fracture toughness.

At a continuum level, it is clear that the plane strain thickness (e.g., sample thickness) and yield strength play dominant roles. It is also apparent from the virtual

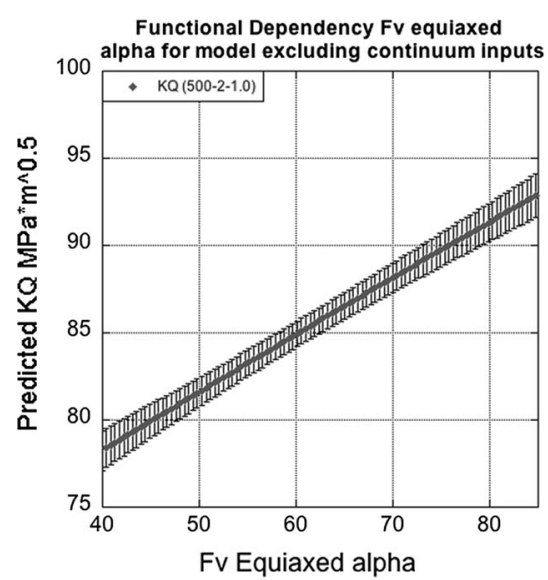

(a)

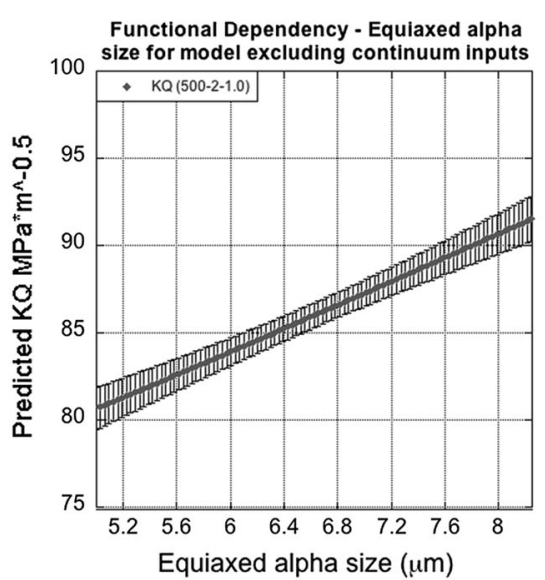

(b)



(c)

Fig. 10-Result of virtual experiments: $(a$ through $c$ ) The influence of microstructural variables on fracture toughness excluding the influence of yield strength. 


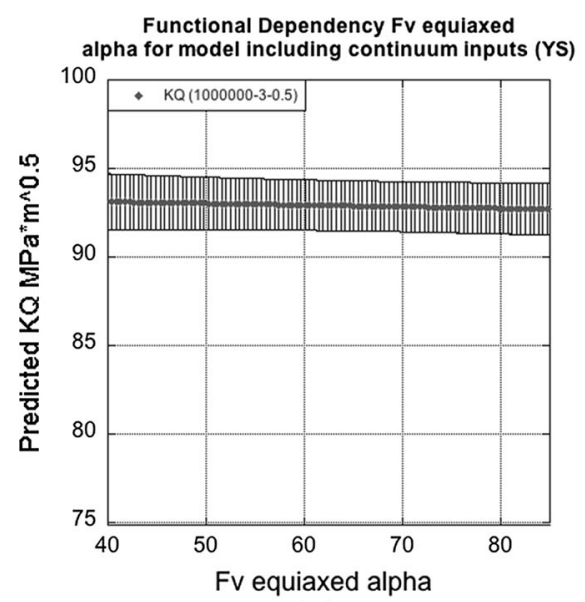

(a)

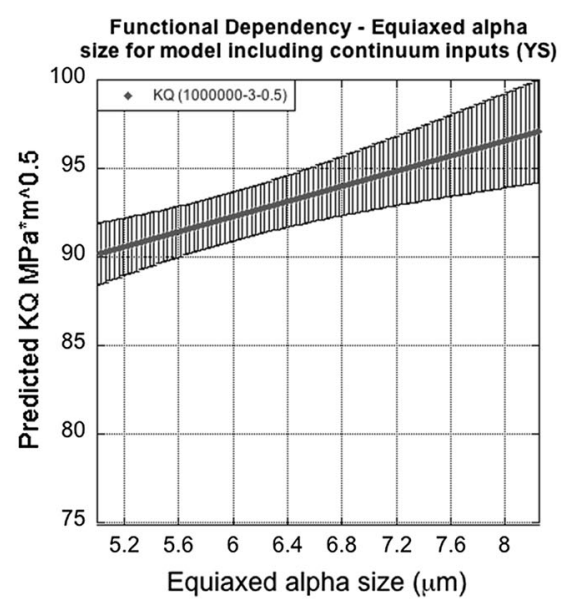

(b)

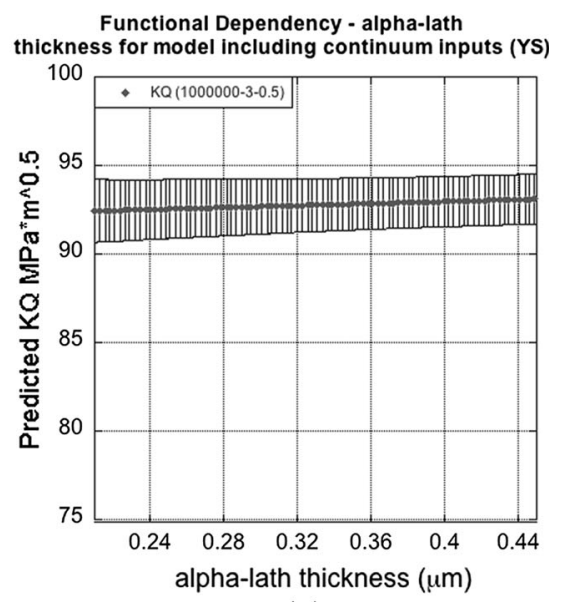

(c)

Fig. 11-Result of virtual experiments: $(a$ through $c$ ) The influence of microstructural variables on fracture toughness for models including yield strength.

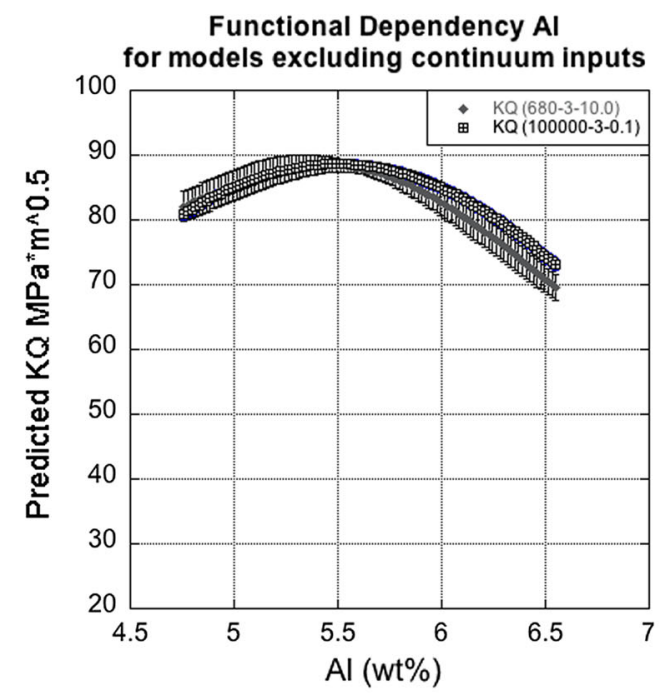

(a)

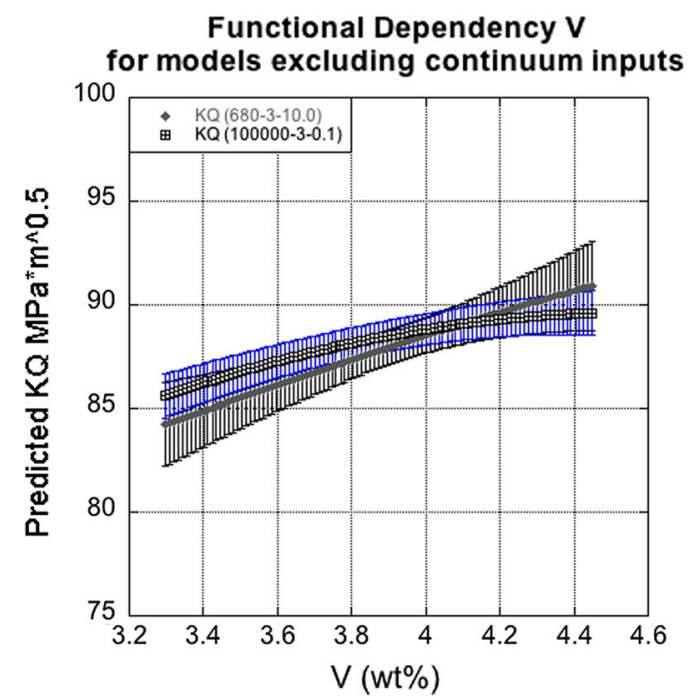

(c)

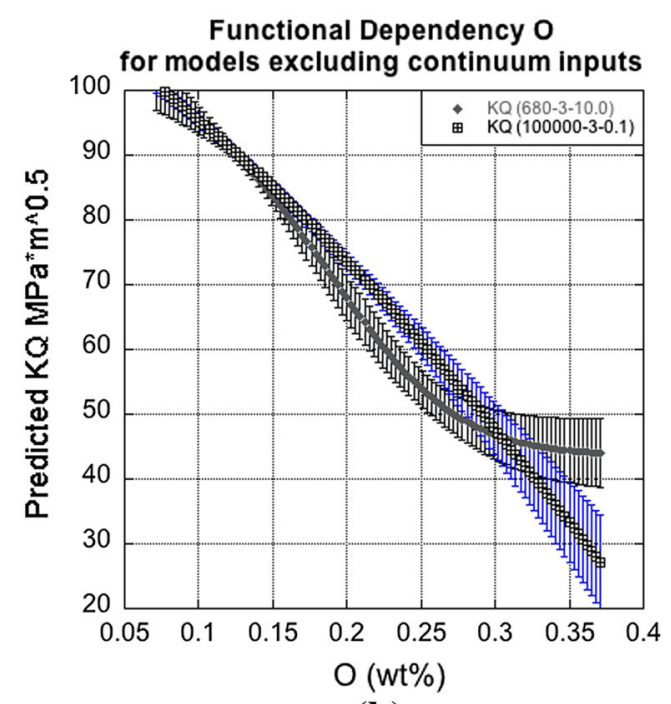

(b)

Functional Dependency Fe for models excluding continuum inputs

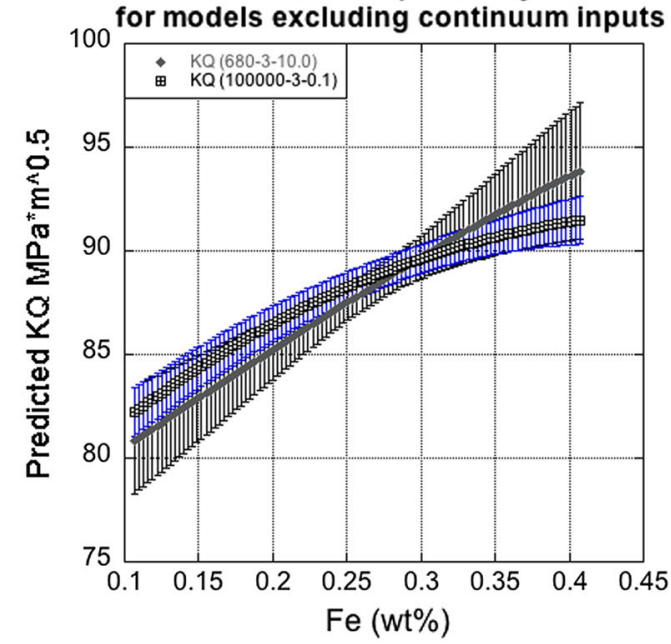

(d)

Fig. 12-Result of virtual experiments: $(a$ through $d$ ) The influence of compositional variables on fracture toughness excluding the influence of yield strength. 
Functional Dependency Al for models including continuum inputs (YS)

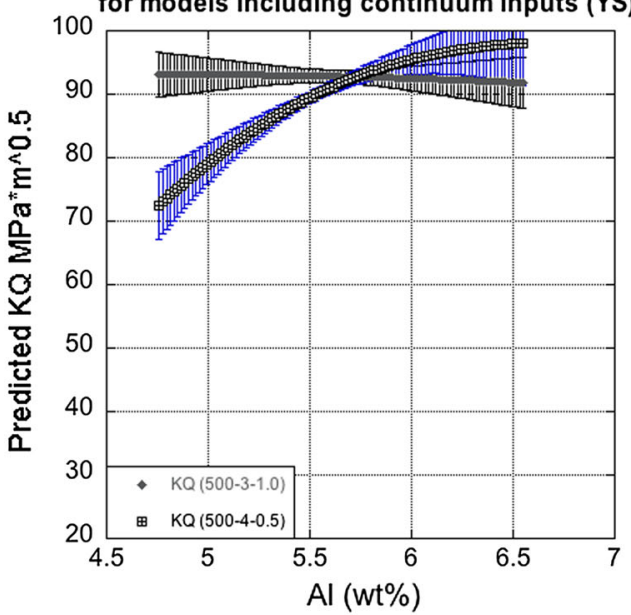

(a)

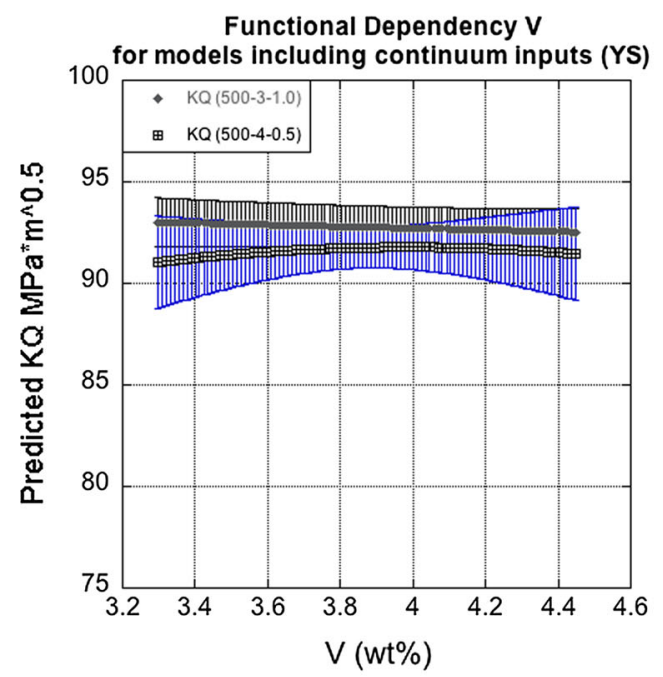

(c)



(b)

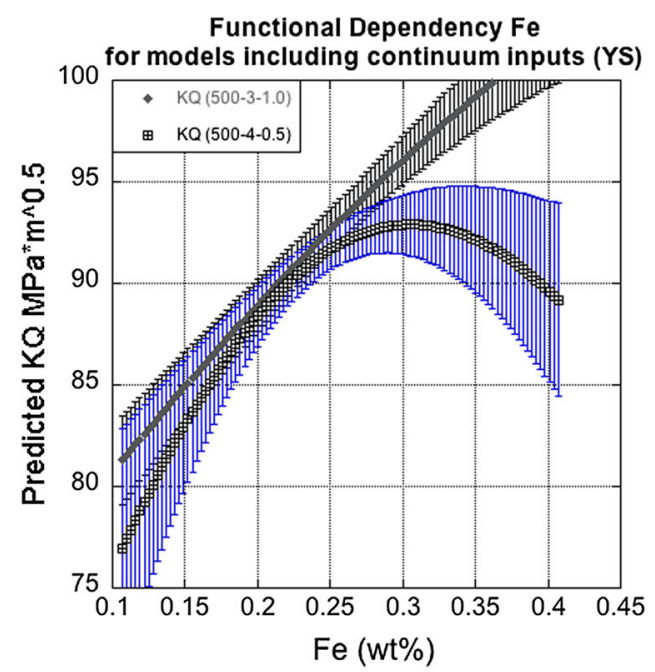

(d)

Fig. 13-Result of virtual experiments: $(a$ through $d$ ) The influence of compositional variables on fracture toughness including the influence of yield strength.

experiments for models including and excluding yield strength that the thickness of the alpha laths and the volume fraction of the equiaxed alpha particles, while directly influencing the yield strength of the material (and hence its resistance to the crack tip opening), do not directly influence the toughness. Further, the size of the equiaxed alpha particles, while influencing the yield strength, also directly contributes to fracture toughness through micromechanistic details. Lastly, it is possible to estimate the effect of plane strain thickness of toughness values that do not satisfy the strict $\boldsymbol{K}_{\mathbf{1 C}}$ requirements. Indeed, $\boldsymbol{K}_{\boldsymbol{Q}}$ may be as much as $10 \mathrm{pct}$ greater than a corresponding $\boldsymbol{K}_{\mathbf{1} \boldsymbol{C}}$ would be. This estimation represents a powerful use of the neural network approach, and may provide a quantitative tool for components limited by fracture toughness.

The development of an approach whereby different architectures are compared has made it possible to gain new insights into fracture toughness in a two-phase ductile material. Such experimental studies are rarely conducted on ductile metallic systems such as $\sigma+\beta$ processed $\mathrm{Ti}-6 \mathrm{Al}-4 \mathrm{~V}$. It is expected that a similar comparison of multiple neural network architectures may lead to new interpretations of critical microstructural features for similarly complex mechanical properties in other multi-component, multi-phase engineering alloys.

\section{ACKNOWLEDGMENTS}

This paper reflects work conducted when all authors were at The Ohio State University. The authors would like to gratefully acknowledge the support provided by the Office of Naval Research, Program \# 2626753 Direct-3-Dimensional (D-3D), Dr. Julie Christodoulou, Program Manager. The authors would also like to 
acknowledge the Metals Affordability Initiative (LAD-2) for the property and toughness data. The authors would also like to thank Dr. Babu Viswanathan for invaluable technical discussions. The views, opinions, and/or findings expressed are those of the authors and should not be interpreted as representing the official views or policies of the Department of Defense or the U.S. Government.

\section{REFERENCES}

1. S Kar, T Searles, E Lee, GB Viswanathan, HL Fraser, J Tiley, and R Banerjee: Metall. Mater. Trans. A, 2006, vol. 37, pp. 559-66.

2. PC Collins, CV Haden, I Ghamarian, BJ Hayes, T Ales, G Penso, V Dixit, and G Harlow: JOM, 2014, vol. 66, pp. 1299-1309.

3. I Ghamarian, P Samimi, V Dixit, and PC Collins: Metall. Mater. Trans. A, 2015, vol. 46, pp. 5021-37.

4. I Ghamarian, B Hayes, P Samimi, BA Welk, HL Fraser, and PC Collins: Mater. Sci. Eng., A, 2016, vol. 660, pp. 172-80.

5. Y Sun, W Zeng, Y Han, X Ma, Y Zhao, P Guo, G Wang, and MS Dargusch: Comput. Mater. Sci., 2012, vol. 60, pp. 239-44.

6. JW Foltz, B Welk, PC Collins, HL Fraser, and JC Williams: Metall. Mater. Trans. A, 2011, vol. 42, pp. 645-50.

7. $\mathrm{H} \mathrm{Li}, \mathrm{DE}$ Mason, Y Yang, TR Bieler, MA Crimp, and CJ Boehlert: Philos. Mag., 2013, vol. 93, pp. 2875-95.

8. JR Seal, MA Crimp, TR Bieler, and CJ Boehlert: Mater. Sci. Eng., $A$, 2012, vol. 552, pp. 61-8.

9. H Li, CJ Boehlert, TR Bieler, and MA Crimp: Philos. Mag., 2015, vol. 95 , pp. 691-729.

10. N Kotkunde, HN Krishnamurthy, P Puranik, AK Gupta, and SK Singh: Mater. Des., 2014, vol. 54, pp. 96-103.
11. B Barkia, V Doquet, JP Couzinié, I Guillot, and E Héripré: Mater. Sci. Eng., A, 2015, vol. 636, pp. 91-102.

12. A. Rosenfield and A.J. McEvily, Jr: AGARD Report 610, NATO Advisory Group for Aerospace Research and Development, December 1973, p. 23-55.

13. RO Ritchie and AW Thompson: Metall. Trans. A, 1985, vol. 16, pp. 233-48.

14. S Osovski, A Srivastava, L Ponson, E Bouchaud, V Tvergaard, K Ravi-Chandar, and A Needleman: J. Mech. Phys. Solids, 2015, vol. 76 , pp. $20-46$.

15. L. Ponson, A. Srivastava, S. Osovski, E. Bouchaud, V. Tvergaard, and A. Needleman: arXiv preprint arXiv:1307.4413, 2013.

16. G Lütjering: Mater. Sci. Eng., A, 1999, vol. 263, pp. 117-26.

17. H. Margolin, P.A. Farrar, and M.A. Greenfield: in The Science, Technology and Application of Titanium, R.I Jaffee and N.E. Promisel, eds., Pergamon, NY, 1970, pp. 795-808.

18. M Niinomi and T Kobayashi: Mater. Sci. Eng., A, 1996, vol. 213, pp. 16-24.

19. NL Richards: J. Mater. Eng. Perform., 2004, vol. 13, pp. 218-25.

20. PC Collins, S Koduri, B Welk, J Tiley, and HL Fraser: Metall. Mater. Trans. A, 2013, vol. 44, pp. 1441-53.

21. D.J.C. MacKay: PhD Thesis, 1992, California Inst. of Tech., Pasadena, CA.

22. DJC Mackay: Neural Computation, 1992, vol. 4, pp. 448-72, https://doi.org/10.1162/neco.1992.4.3.415.

23. PC Collins, B Welk, T Searles, J Tiley, JC Russ, and HL Fraser: Mater. Sci. Eng., A, 2009, vol. 508, pp. 174-82.

24. Y Liu, P Samimi, I Ghamarian, DA Brice, DE Huber, Z Wang, V Dixit, S Koduri, HL Fraser, and PC Collins: JOM, 2015, vol. 67, pp. 164-78.

25. R. Boyer, E.W. Collings, and G. Welsch (editors), Materials Properties Handbook: Titanium Alloys, ASM International, 1994, p. 581.

26. S.K. Kar: PhD Thesis, 2005, The Ohio State University, Columbus, $\mathrm{OH}$. 